\title{
rFVIla transported from blood stream into tissues is functionally active
}

\author{
R. GOPALAKRISHNAN ${ }^{\star}$, U. HEDNER ${ }^{\dagger}$, C. CLARK ${ }^{*}$, U.R. PENDURTHI ${ }^{*}$, and L.V.M RAO ${ }^{*}$ \\ "Center for Biomedical Research, The University of Texas Health Science Center, Tyler, TX, USA \\ †Department of Medicine, Malmö University Hospital, University of Lund, Malmö, Sweden
}

\begin{abstract}
Recombinant factor VIIa (rFVIIa) is used widely as an effective hemostatic agent for treatment of severe hemophilia patients with inhibitors against FVIII or FIX. Recent reports have shown that rFVIIa when administered in prophylaxis can prevent the development of mild-moderate joint bleedings [1-7]. Given the short biological half-life of rFVIIa, it is unclear by what mechanism rFVIIa could be effective in prophylactic treatment. A recent study characterizing the bio-distribution of pharmacologically administered rFVIIa by immunohistochemistry revealed that rFVIIa (1) readily associates with the endothelium, (2) enters in to extravascular tissues where it can bind to TF, and (3) remain there for longer time periods than in the circulation [8]. Although these immunohistochemistry data provide convincing evidence that rFVIIa from the blood stream enters into extravascular compartments, they provide no quantification of the amount of rFVIIa transferred from the blood stream to extravascular tissues, and no information regarding the functional status of rFVIIa associated with these tissues. Moreover, recent studies of Hoffman et al. [9] indicated that perivascular TF is occupied by endogenous FVII/FVIIa in the absence of injury. In such scenario, the exogenously administered rFVIIa may be simply supplanting the endogenous FVII/FVIIa bound to peri/extravascular tissues without increasing the net levels of FVIIa in tissues. Therefore, we thought it was important to evaluate FVIIa levels in tissues following its administration in order to assess the extent of rFVIIa transfer from the blood stream into tissues, and the functional status of rFVIIa associated with extravascular tissues.
\end{abstract}

First, to determine whether TF in perivascular tissue is saturated with endogenous FVII/ FVIIa, we have examined the presence of mouse FVII in adventitia of blood vessels from mice receiving saline by immunohistochemistry using polyclonal antibodies specific to mouse FVII/FVIIa. The antibodies were raised in rabbits using purified recombinant mouse rFVIIa as the antigen. The antibodies detect both mouse FVII and FVIIa. Out of 25 or more tissue sections of skin, liver and heart that we have examined, positive immunostaining of FVII was found only once around a single blood vessel in skin (data not shown). None of the other blood vessels or tissues stained positive for FVII/FVIIa. It is unclear why our immunohistochemistry data differs from that of Hoffman et al. [9]. It is possible that different mouse FVIIa antibodies used in these studies could have contributed to this difference. Although our present data suggest that it is unlikely that the peri or extravascular

\footnotetext{
Address correspondence to: L. Vijaya Mohan Rao, Ph.D. Department of Biochemistry Center for Biomedical Research The University of Texas Health Science Center at Tyler 11937 US Highway 271 Tyler, TX 75703 Tel: (903)877-7332 Fax: (903)877-7426 vijay.rao@uthct.edu.

Addendum R. Gopalakrishnan performed the experiments and FVII activity assays. C. Clark performed FVIIa activity and FVII/VIIa antigen assays. Both Gopalakrishnan and Clark analyzed the data. U. Hedner and U.R. Pendurthi participated in the research design, reviewing data and preparation of the manuscript. L.V.M. Rao designed and reviewed the research, analyzed the data and wrote the manuscript.
} 
TF is saturated with endogenous FVII, we cannot rule out the possibility that mouse FVIIa antibodies used in this study may not have sufficient sensitivity to detect traces of FVII/ FVIIa in tissues. Therefore, in additional studies we have examined whether exogenously added FVIIa binds to tissue sections. Skin and other tissue sections were incubated with mFVIIa $(10 \mathrm{nM}), \mathrm{CaCl}_{2}(5 \mathrm{mM})$, or $\mathrm{mFVIIa}+\mathrm{CaCl}_{2}$ for $1 \mathrm{~h}$, washed and then stained with anti-mFVIIa. Only tissue sections incubated with mFVIIa in the presence of calcium ions stained positively, whereas other tissue sections stained negatively for FVIIa (data not shown). These data indicate that exogenously added FVIIa is capable of binding to extravascular TF, suggesting that TF sites are not saturated with endogenous FVII.

To determine the extent of rFVIIa transported to tissues following its administration into the blood stream, mice were injected intravenously with saline or mFVIIa $(120 \mu \mathrm{g} / \mathrm{kg}$ body weight, $\mathrm{n}=3$ to 4 ) via tail vein. Thirty minutes, $6 \mathrm{~h}$ and $24 \mathrm{~h}$ post-administration of rFVIIa (and $30 \mathrm{~min}$ post-administration of saline), mice were exsanguinated by flushing $10 \mathrm{ml}$ of ice-cold saline (supplemented with $\mathrm{CaCl}_{2}, 5 \mathrm{mM}$ ) through the heart and draining the blood by severing the renal artery. Various tissues (e.g., lung, liver, kidney, brain, spleen, heart and skin) were collected and stored at $-80^{\circ} \mathrm{C}$ until homogenization. The tissues were weighed and homogenized in ice-cold TBS $(0.01 \mathrm{M}$ Tris- $\mathrm{HCl}, 0.15 \mathrm{M} \mathrm{NaCl}, \mathrm{pH} 7.4,0.5 \mathrm{ml} / 100 \mathrm{mg}$ tissue). Initially, we measured FVII/FVIIa activity in total tissue homogenates as well as supernatants of tissue homogenates after the addition of EDTA $(20 \mathrm{mM})$ in factor $\mathrm{X}$ activation assay using saturating concentrations of relipidated TF $(100 \mathrm{ng} / \mathrm{ml})$. We obtained similar levels of FVII/FVIIa activity in both tissue homogenates and the supernatants. FVII/ FVIIa activity levels were higher in tissues derived from mice receiving rFVIIa compared to mice receiving saline. These data suggest that rFVIIa administered to mice entered into tissues and remained functionally active. Based on this information, we have used the EDTA supernatants for all of our subsequent assays. We have chosen the supernatant over the tissue homogenates for measuring FVIIa levels because varying levels of TF present in different tissue extracts may introduce error in determining FVIIa levels accurately. More importantly, presence of cell-derived full-length TF does not permit measurement of FVIIa activity in FVIIa-specific clotting assay using soluble TF [10]. Furthermore, it is not feasible to measure FVIIa antigen accurately in tissue homogenates if it is bound to TF or other cellassociated proteins.

Measurement of FVIIa activity in tissues using FVIIa-specific clotting assay [10] from saline administered mice showed that all tissues contained traces, but measurable amount of FVIIa, varying from 0.1 to $1 \mathrm{ng} / \mathrm{mg}$ tissue protein. Following the administration of rFVIIa, FVIIa levels increased in most of the tissues (Fig. 1A). The extent of increase in FVIIa in different tissues varied. FVIIa levels increased by 5 to 10 -fold in heart, spleen, kidney, liver and lung. The increase in FVIIa in tissues was highest at 30 min following the administration of rFVIIa. In some tissues, FVIIa levels remained high (similar to that were found at $30 \mathrm{~min}$ ) even at $24 \mathrm{~h}$ following rFVIIa administration, at which time rFVIIa in plasma was undetectable. No detectable increase in FVIIa was found in brain and skin at any time following the infusion of rFVIIa. The well-recognized property of the blood-brain barrier may be responsible for curtailing the transport of FVIIa from blood to brain. In addition to measuring rFVIIa activity, we also measured FVII/FVIIa antigen in tissue extracts using ELISA specific for mouse FVII/FVIIa. In saline administered mice, FVII/ FVIIa antigen levels in various tissues varied from 1 to $6 \mathrm{ng} / \mathrm{mg}$ protein (Fig. 1B), which is $\sim 6$ to 10-fold higher than FVIIa levels determined in FVIIa specific clotting assay. This indicates that most of the endogenous FVII associated with the tissues remains as the zymogen and not activated. Similar to that observed in FVIIa activity measurements, FVII/ FVIIa antigen in tissues of mice were significantly higher following rFVIIa administration. The increase in FVII antigen was evident at $30 \mathrm{~min}$ and $6 \mathrm{~h}$ post of rFVIIa administration (Fig. 1B). However, the fold-increase of FVII/FVIIa measured by estimating the antigen 
levels in tissues following rFVIIa administration was much lower than that was estimated using FVIIa specific clotting activity assay. A significant increase in FVII antigen was not found in tissues harvested at $24 \mathrm{~h}$ following rFVIIa administration. Here, it is important to note that, unlike FVIIa specific clotting activity assay, the antigen assay measures both FVII and FVIIa, thus a small yet potentially significant increase in FVIIa activity following rFVIIa administration may be difficult to detect relative to total FVII/FVIIa antigen present endogenously. While it is unlikely that endogenous FVII/FVIIa detected in tissues is plasma protein remaining following perfusion of the animal as we adequately perfused the animal and no breach in vascular endothelium was evident (visualized by EPCR immunohistochemistry), we can not exclude this possibility completely.

Although from the above data it may appear that only traces of rFVIIa administered to mice enters into extravasculature and is retained in tissues, it is important to compare the levels of FVIIa in tissues in relation to FVIIa levels in plasma following rFVIIa administration to appreciate the significance of FVIIa levels in tissues. As shown in Fig. 1C, 30 min following rFVIIa administration, most of the injected rFVIIa in mice was in plasma and only about $4 \%$ of rFVIIa was in tissues, mostly in liver. Six $\mathrm{h}$ following rFVIIa administration, the majority of rFVIIa found in mice was in tissues. At $24 \mathrm{~h}$, all of the rFVIIa detected in mice was in tissues and none in plasma. This clearly illustrates that rFVIIa distributed to extravasculature is retained much longer than rFVIIa in circulation.

Overall, the quantitative determination of FVIIa activity and antigen in various tissues in mice following rFVIIa administration is consistent with our recent findings of biodistribution of rFVIIa by immunohistochemistry [8] in that rFVIIa administered i.v. enters into extravascular tissues and FVIIa is retained in tissues for a much longer time compared to plasma levels. However, the data obtained in the present study differ with the earlier data in two aspects. First, in the earlier study [8], we could not detect rFVIIa in lung tissue sections by immunohistochemistry whereas in the present study we found a significant increase in both FVIIa activity and antigen levels in lung tissue following rFVIIa administration. It is possible that diffused distribution of rFVIIa throughout the lung could have escaped detection by immunohistochemistry. Second, in the earlier study we found rFVIIa accumulation in skin by immunohistochemistry, particularly in cuboidal epithelial cells of the sebaceous glands adjacent hair follicles and in the squamous epithelial cells of the epidermis. In the present study we did not detect any significant increase in FVIIa activity and antigen in skin homogenates. However, it may be pertinent to point out here that it was difficult to homogenize skin tissues as it is elastic and consistently resulted in a very viscous and glue-like mixture. It is possible that FVIIa associated with skin is readily internalized and degraded, yet this degraded FVIIa could still be detected by AF488 antibodies in immunohistochemistry. It is also possible that differences in tissue densities could have contributed to this anomaly.

At present, it is unclear whether or how FVIIa associated with extravascular tissue contributes to the hemostatic and prophylactic effects of rFVIIa. In this context, it is interesting to point out that a recent gene therapy study in hemophilia B dogs showed that one of the hemophilia B dogs, which had no significant increase in circulating levels of FVIIa, still did not develop any spontaneous bleedings during the 34 months of observation [11]. These findings support the hypothesis that rFVIIa deposited perivascularly prevents joint bleeding in hemophilia by stopping early bleeding in the microvasculature induced by normal moving patterns. 


\section{Acknowledgments}

The authors are thankful to Novo Nordisk, Denmark for providing funding for this project. This work was supported partly by National Institutes of Health grants HL58869 and HL65500. The authors are thankful to Mirella Ezban and Lars C. Petersen, Novo Nordisk, Denmark, for providing mouse rFVIIa.

Disclosure and conflict of interests The study was supported primarily by a research grant from Novo Nordisk, Denmark. U. Hedner is a consultant to Novo Nordisk A/S, Zurich, Switzerland.

\section{References}

1. Saxon BR, Shanks D, Jory CB, Williams V. Effective prophylaxis with daily recombinant factor VIIa (rFVIIa-Novoseven) in a child with high titre inhibitors and a target joint. Thromb Haemost. 2001; 86:1126-7. [PubMed: 11686341]

2. Young G, McDaniel M, Nugent DJ. Prophylactic recombinant factor VIIa in haemophilia patients with inhibitors. Haemophilia. 2005; 11:203-7. [PubMed: 15876264]

3. Blatny J, Kohlerova S, Zapletal O, Fiamoli V, Penka M, Smith O. Prophylaxis with recombinant factor VIIa for the management of bleeding episodes during immune tolerance treatment in a boy with severe haemophilia A and high-response inhibitors. Haemophilia. 2008

4. Morfini M, Auerswald G, Kobelt RA, Rivolta GF, Rodriguez-Martorell J, Scaraggi FA, Altisent C, Blatny J, Borel-Derlon A, Rossi V. Prophylactic treatment of haemophilia patients with inhibitors: clinical experience with recombinant factor VIIa in European Haemophilia Centres. Haemophilia. 2007; 13:502-7. [PubMed: 17880436]

5. Tcheng WY, Donkin J, Konzal S, Wong WY. Recombinant factor VIIa prophylaxis in a patient with severe congenital factor VII deficiency. Haemophilia. 2004; 10:295-8. [PubMed: 15086331]

6. Mathijssen NC, Masereeuw R, Verbeek K, Lavergne JM, Costa JM, van Heerde WL, Novakova IR. Prophylactic effect of recombinant factor VIIa in factor VII deficient patients. Br J Haematol. 2004; 125:494-9. [PubMed: 15142120]

7. Konkle BA, Ebbesen LS, Erhardtsen E, Bianco RP, Lissitchkov T, Rusen L, Serban MA. Randomized, prospective clinical trial of recombinant factor VIIa for secondary prophylaxis in hemophilia patients with inhibitors. J Thromb Haemost. 2007; 5:1904-13. [PubMed: 17723130]

8. Gopalakrishnan R, Hedner U, Ghosh S, Nayak R, Allen TC, Pendurthi UR, Rao LV. Biodistribution of pharmacologically administered rFVIIa. J Thromb Haemost. 2010; 8:301-10. [PubMed: 19943873]

9. Hoffman M, Colina CM, McDonald AG, Arepally GM, Pedersen L, Monroe DM. Tissue factor around dermal vessels has bound factor VII in the absence of injury. J Thromb Haemost. 2007; 5:1403-8. [PubMed: 17425666]

10. Morrissey JH, Macik BG, Neuenschwander PF, Comp PC. Quantitation of activated factor VII levels in plasma using a tissue factor mutant selectively deficient in promoting factor VII activation. Blood. 1993; 81:734-44. [PubMed: 8427965]

11. Margaritis P, Roy E, Aljamali MN, Downey HD, Giger U, Zhou S, Merricks E, Dillow A, Ezban M, Nichols TC, High KA. Successful treatment of canine hemophilia by continuous expression of canine FVIIa. Blood. 2009; 113:3682-9. [PubMed: 19109232] 


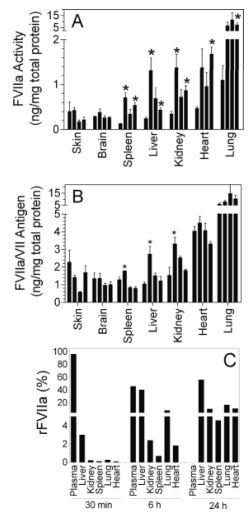

Fig. 1.

FVIIa activity and antigen in tissues following rFVIIa administration. Mouse rFVIIa (120 $\mu \mathrm{g} / \mathrm{kg}$ body weight in $100 \mu \mathrm{l}$ ) or saline was administered i.v. to C57BL/6 mice. Mice were exsanguinated at $30 \mathrm{~min}, 6 \mathrm{~h}$, and $24 \mathrm{~h}$ following the administration of rFVIIa. Various tissues were collected and homogenized (500 $\mu \mathrm{l} / 100 \mathrm{mg}$ tissue) using tissue homogenizer. Tissue homogenates were centrifuged (after adding EDTA, $20 \mathrm{mM}$ ) and the supernatants were collected and assayed for FVIIa levels in FVIIa-specific clotting activity assay using soluble TF (A) or FVII/FVIIa antigen in ELISA using antibodies specific to mouse FVII/ FVIIa (B). For the clotting assay, the samples were diluted at least 10 times in TBS supplemented with $1 \mathrm{mg} / \mathrm{ml}$ bovine serum albumin before they were used in the assay. Under these conditions, EDTA will not affect the assay. Protein concentrations in tissue supernatants were measured using Bio-Rad protein assay kit. FVIIa activity and antigen levels were normalized to the protein concentration. The four bars in each group in panels A and $\mathrm{B}$ represent FVIIa levels in mice at $30 \mathrm{~min}$ following saline administration or $30 \mathrm{~min}, 6$ h, $1 \mathrm{~d}$ following rFVIIa administration, respectively. *indicates statistically significant increase in FVIIa activity or antigen compared to the value obtained in the corresponding tissue of mice administered with saline. Panel C depicts the amount of rFVIIa (in \%) in plasma and various organs following rFVIIa administration. To determine FVIIa levels specific to rFVIIa administration, FVIIa activity levels measured in saline-administered mice were deducted from FVIIa activity levels measured following rFVIIa administration. rFVIIa associated in each organ was determined by taking into consideration of organ weight and the amount of protein in the organ. rFVIIa activity found in plasma, liver, kidney, brain, lung, heart and spleen at specific time periods were added together and the combined value was taken as $100 \%$ to determine fraction of FVIIa associated with each tissue at a specific time point. 